\title{
Atomic Ordering in the Liquid Adjacent to an Atomically Rough Solid Surface
}

\author{
B. Jiang, H. Men and Z. Fan* \\ BCAST, Brunel University London, Uxbridge, Middlesex, UB8 3PH, UK. \\ *Corresponding author. Tel.: +44 1895 266406; Fax: +44 1895 269758; E-mail \\ address: Zhongyun.Fan@brunel.ac.uk
}

\begin{abstract}
In this work, atomic ordering in the liquid adjacent to both crystalline and amorphous substrates with atomic level surface roughness was investigated systematically using molecular dynamics (MD) simulations. We found for the first time that increasing surface roughness of a crystalline substrate reduces both atomic layering and in-plane atomic ordering in the metallic liquid adjacent to the liquid/substrate interface. In addition, our MD simulation results revealed that the rough surface of an amorphous substrate eliminates completely in-plane ordering in the liquid regardless of surface roughness and reduces/eliminates atomic layering in the liquid depending on the level of surface roughness. This reduced atomic ordering in the liquid adjacent to an atomically rough surface can be attributed to the increase in mobility of atoms in the liquid compared with the case with a smooth crystalline surface. From the point of view of heterogeneous nucleation, in addition to the effect of lattice misfit investigated in our previous studies, this work provides further confirmation of the importance of structural templating as a mechanism for both prenucleation and heterogeneous nucleation. Furthermore, this work offers a new approach to impede heterogeneous nucleation by roughening the substrate surface at the atomic level.
\end{abstract}

Keywords: Atomic ordering; Interface; Surface roughness; MD simulation

\section{Introduction}

Atomic ordering in the liquid adjacent to a solid substrate has recently attracted increasing interest in the solidification research community, due to its implications for heterogeneous nucleation [1,2]. Such atomic ordering at temperatures above the liquidus has been referred to as prenucleation [3], which can be taken as a precursor for the subsequent heterogeneous nucleation process. The Epitaxial Nucleation model [4] suggests that heterogeneous nucleation proceeds through layer-by-layer growth by 
a structural templating mechanism. The crystal lattice in the substrate surface provides low energy positions where the adjacent liquid atoms can form a locally ordered structure, which in turn templates the formation of an ordered structure in the next layer and so on. The undercooling required for epitaxial nucleation is closely related to the compatibility of the crystal structures of the substrate and the solidified phase, which can be quantified by their lattice misfit. Therefore, one would expect that pronounced atomic ordering in the liquid at the interface, above the liquidus, can have a significant influence on the heterogeneous nucleation process. If atomic ordering at the interface is compatible with the crystal structure of the solid it would enhance heterogeneous nucleation by reducing the nucleation barrier; otherwise, incompatible atomic ordering at the interface would impede heterogeneous nucleation. Therefore, it is important, both scientifically and technologically, to have a good understanding of how the chemical and/or physical properties of the substrate affect atomic ordering in the liquid at the interface and its implications for the heterogeneous nucleation process.

Both experimental observations [5-10] and atomistic simulations [11-16] have been conducted to understand atomic ordering in the liquid at liquid/substrate interfaces. These studies suggest that at temperatures above the liquidus the liquid atoms become layered within one or two nanometres of the interface (atomic layering) and that the atoms in individual atomic layer may have a substantially ordered structure (in-plane atomic ordering). Oh et al. $[9,10]$ have provided firm evidence for atomic layering and in-plane ordering in liquid $\mathrm{Al}$ adjacent to $\alpha-\mathrm{Al}_{2} \mathrm{O}_{3}$ substrates with a [0001] surface orientation, through in situ observation by high-resolution transmission electron microscopy (HRTEM). In their MD simulations with adapted n-body potentials, Geyermans et al. [11] revealed that a solid $\mathrm{Cu}$ wall induces significant layering in the liquid $\mathrm{Al}$ at the interface, largely independent of surface orientation of the substrates. Using a semi-empirical potential of an embedded-atom method (EAM), Hashibon et al. $[12,13]$ revealed an exponential decay of density profile in the liquid Al at the interface, and found that there is far greater in-plane ordering in the liquid in contact with a bcc (100) substrate than that in contrast with a bcc (110) substrate. These atomistic simulations offer access to microscopic details of atomic ordering in the liquid adjacent to the liquid/solid interface.

The atomic ordering in a given liquid at the interface can be manipulated by changing the structure and/or chemistry of the substrate. The layering has been attributed to the 'hard wall' effect of the substrate surface [17], and theoretical calculations [18] suggest that the liquid has an oscillatory density profile at the interface with a structureless solid wall. The degree of the layering is usually independent of crystal structure [12], surface orientation [11,12] of a substrate with a smooth surface, and lattice misfit between the substrate and the solid phase corresponding to the liquid [14]. Atomic layering has even been observed in metallic liquids adjacent to their own surfaces by x-ray reflectivity measurements [17,19-21], and at the interface with the 
smooth surface of an amorphous substrate using the MD simulation [11]. All these studies suggest that the layering at the interface hardly changes by changing the substrates as long as the substrate surface is smooth. On the other hand, the in-plane atomic ordering at the interface has been attributed to the crystalline lattice in the surface of the substrate, which provides potential low energy positions for the liquid atoms at the interface. Therefore, the in-plane atomic ordering is closely related to the crystal structure of the substrate [11-14]. Using MD simulations, it is found that the in-plane ordering persists within the first 3 atomic layers adjacent to an interface having a small lattice misfit, and becomes very weak, even in the $1^{\text {st }}$ layer for substrates having a large lattice misfit [14]. This suggests that the in-plane atomic ordering can be manipulated by changing the crystallographic matching between the substrate and the solid upon solidification. In addition, we found recently that chemical interactions between the substrate and the liquid may further enhance or impede the structural effect on atomic ordering at the interface, including both layering and in-plane ordering [22].

The atomic ordering in the liquid at the liquid/substrate interface may be affected by the surface roughness of the substrate. To date, only a small number of studies on this topic have been reported in the literature. Using MD simulations, Geysermans et al. [11] revealed that atomic layering is significantly weakened by increasing the surface roughness of an amorphous substrate, and even destroyed completely by the rough surface of a bulk amorphous substrate. Galea et al. [23] investigated the effect of atomic level roughness of crystalline substrates on slip length at the fluid/solid boundary during shear flow, by varying the size and spacing of substrate atoms at a constant packing fraction, and they found that the amplitude of the density oscillations at the interface increases with increasing smoothness of the surfaces. In both cases, however, the effect of surface roughness on the in-plane atomic ordering was not investigated. Therefore, it is desirable to clarify how atomic ordering (both layering and in-plane ordering) in the liquid at the interface is affected by the surface roughness of the substrate.

This study aims to investigate systematically the effect of atomic level surface roughness of the substrate on atomic ordering in the liquid adjacent to the liquid/substrate interface, using MD simulations.

\section{Atomic level surface roughness}

Here we define atomic level surface roughness, $R$, by the following equation:

$$
R=\frac{\Delta h}{h} \times 100 \%
$$

where $\Delta h$ is the maximum distance of surface atoms away from a smooth reference plane; $h$ is the spacing of the smooth reference plane, and $\Delta h<h$. For instance, the 
atomic level surface roughness of a $\{111\}$ terminated surface of an fcc crystal is $0 \%$. In this case the smooth reference plane is the $\{111\}$ plane, and $h$ is the $\{111\}$ plane spacing, $d\{111\}$.

In order to investigate the effect of atomic level surface roughness on atomic ordering in the liquid, we artificially construct an atomic level rough surface from an fcc substrate with a [111] surface orientation (Fig. 1). Our starting point is a single (111) plane of fcc structure, in which the atoms are arranged in a hexagonal pattern. To vary surface roughness, while atomic positions of the [110] atomic row in one of every $p^{\text {th }}$ row remains unchanged (dark spheres in the $i^{\text {th }}=1$ row for $p=3$ in Fig. 1(a)), the atoms in the $i^{\text {th }}$ atomic rows (light spheres with solid border) are displaced by a distance of $(i-1) \Delta h /(p-1)$ along the [111] direction from its original position (light atoms with dashed border) (Fig. 1(b)). Here, $\Delta h$ is the distance of the $p^{\text {th }}$ atomic row displaced away from the (111) plane along the [111] direction, and $h$ is the $\{111\}$ plane spacing, $d_{\{111\}}$. Thus, the atomic level rough surface is created, with a step period of $p$, and the surface roughness of the substrate can be expressed as:

$$
R=\frac{d_{\{112\}}(p-1) \tan \alpha}{d_{\{111\}}} \times 100 \%,
$$

where $d_{\{112\}}$ is the $\{112\}$ plane spacing, and $\alpha$ is an angle defined in Fig. 1(b). Using this procedure, we can artificially create atomic level rough surfaces with varied surface roughness by choosing the right combination of $p$ and $\alpha$.

Eq. (1) is also applicable to the case of surface roughness of an amorphous substrate. In this study, the bulk amorphous solid is prepared by quenching the liquid Al from the equilibration temperature of $1000 \mathrm{~K}$ down to $0 \mathrm{~K}$. A single layer of amorphous solid with a thickness of $d_{\{111\}}$ of fcc Al was taken from the bulk amorphous solid to be the substrate. In order to artificially create a substrate with varying surface roughness, this single layer of the amorphous solid is compressed into varied thickness of $\Delta h$ until $\Delta h$ $=0$, as shown in Fig. 1(c). Thus, the surface roughness, $R$, of the single layer of amorphous substrate can be described by Eq. (1). $R$ is $0 \%$ for $\Delta h=0$ (i.e., $2 \mathrm{D}$ amorphous), and $100 \%$ for the single layer of amorphous substrate with $\Delta h=d_{\{111\}}$ and the bulk amorphous substrate.

\section{Simulation approach}

Both crystalline and amorphous substrates with varied surface roughness are used in the present work. The crystalline substrate has an fcc structure, a [111] surface orientation, a lattice parameter equal to that of $\mathrm{Al}$ at its melting temperature, and a thickness of $6 d\{111\}$. The amorphous substrate is also Al and has a thickness of $6 d\{111\}$ for the case of $R=100 \%$ and $1 d_{\{111\}}$ for other levels of surface roughness. The liquid Al, with a total number of 5000 atoms, is generated by equilibrating the simulation system at $1000 \mathrm{~K}$. During the simulation, the substrate atoms are pinned at their 
equilibrium positions, which eliminate the effects on atomic ordering caused by factors other than surface roughness, such as lattice misfit and chemical interactions.

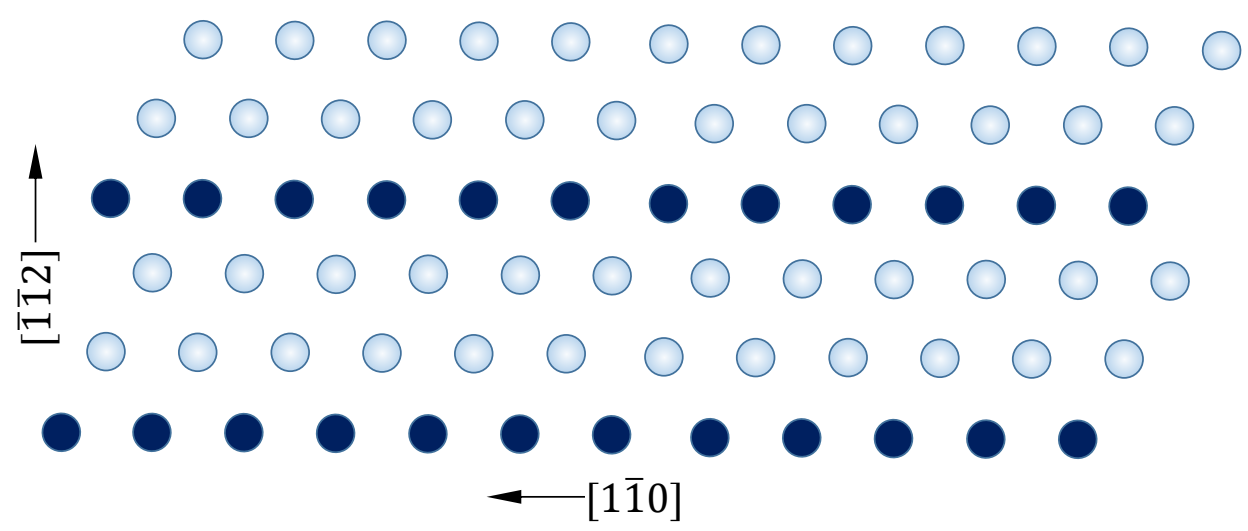

(a)

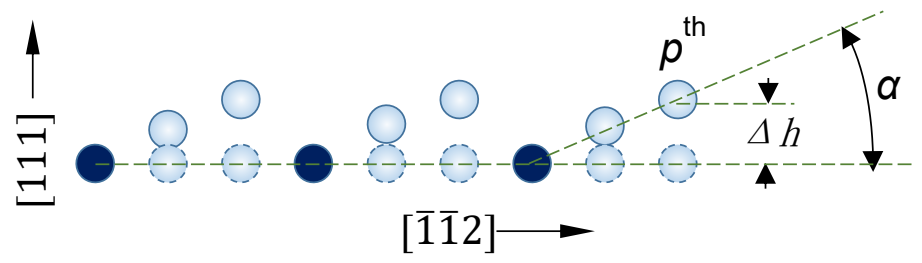

(b)

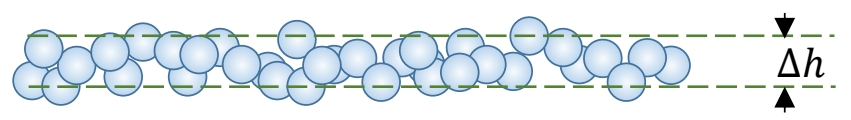

(c)

Fig. 1. Schematic illustration of the artificial construction of substrates with varied atomic level surface roughness. An artificially constructed crystalline rough surface with (111) surface orientation of an fcc Al substrate is viewed (a) from the [111] direction and (b) from the [1ํㅣㄹ direction, and (c) an artificially constructed amorphous Al rough surface is viewed from a direction perpendicular to its surface normal. Dark spheres represent atoms that remain in the original surface plane, and light spheres represent atoms that are displaced in a direction parallel to the surface normal to create surface roughness.

The RGL potential, created by V. Rosato, M. Guillope, and B. Legrand [24], which has been widely employed to simulate metallic systems, was used in the simulation. We use the NVT ensemble, periodic boundary conditions in 3-dimensions and a time step of 1 fs. The simulation usually runs for 500,000 time steps to ensure that the system is equilibrated.

We performed simulations with the RGL potential to validate the simulation approach. The melting temperature was determined to be $916.0 \pm 13.5 \mathrm{~K}$, fairly close to the 
experimental value of $933 \mathrm{~K}$ [26]. The lattice parameter calculated with the NPT ensemble at $298 \mathrm{~K}$ is $4.083 \AA$, which is in good agreement with experimental value of $4.05 \AA[25]$. The calculated elastic constants agree well with the experimental values, as shown in Table 1.

Table 1 Comparison of the physical properties of Al from the simulation in this study with that from experiments in the literature [25-27].

\begin{tabular}{lccc}
\hline & This study & Experiments & Error $(\%)$ \\
\hline Lattice parameter $(\AA)$ & 4.0830 & $4.05[25]$ & 0.83 \\
Melting point $(\mathrm{K})$ & $916.0 \pm 13.5$ & $933.45[26]$ & - \\
$\mathrm{B}_{0}(\mathrm{GPa})$ & 79.07 & $79[27]$ & 0.013 \\
$\mathrm{C} 11(\mathrm{GPa})$ & $113.71 \pm 1.04$ & $114[27]$ & -0.8 \\
$\mathrm{C} 12(\mathrm{GPa})$ & $61.69 \pm 1.39$ & $61.9[27]$ & 0.7 \\
$\mathrm{C} 44(\mathrm{GPa})$ & $31.16 \pm 0.89$ & $31.6[27]$ & -5.2 \\
\hline
\end{tabular}

The atomic ordering in the liquid adjacent to the interface was characterized by the atomic density profile, $\rho(z)$, and in-plane atomic ordering, $S(z)$, and time-averaged atomic positions. $\rho(z)$ is expressed as [12]:

$$
\rho(z)=\frac{\left\langle N_{z}\right\rangle}{L_{x} L_{y} \Delta z}
$$

where $N_{z}$ is the number of atoms between $z-\Delta z / 2$ and $z+\Delta z / 2$ at time $t, \Delta z$ is the width of the bin and the angled brackets indicate a time averaged quantity, and $L_{x}$ and $L_{y}$ are the $x$ and $y$ dimensions of the cell. $S(z)$ is expressed as [28]:

$$
S(z)=\frac{\left|\sum_{j \in \Delta z} \exp \left(i \mathbf{K} \cdot \mathbf{r}_{j}\right)\right|^{2}}{N_{z}^{2}}
$$

where the summation is over all atoms labelled $j$ within a given bin of width $\Delta z, \mathrm{~K}$ is the reciprocal lattice vector, and $\mathbf{r}_{j}$ is the position vector of the $j^{\text {th }}$ atom in Cartesian space. A detailed description of the calculations can be found in Ref. [14].

\section{Results}

\subsection{Effect of surface roughness of crystalline substrates on atomic ordering}

Liquid atoms adjacent to a crystalline substrate with a [111] orientation exhibit a layered structure at $1000 \mathrm{~K}$, as shown by a snapshot of the simulation system in Fig. 2(a). The quantified density profile, $\rho(z)$, as a function of distance, $z$, from the interface is shown in Fig. 2(b). The layering persists within 5 atomic layers at the interface, and the peak density of the individual layer decreases with increasing distance $z$ from the interface.

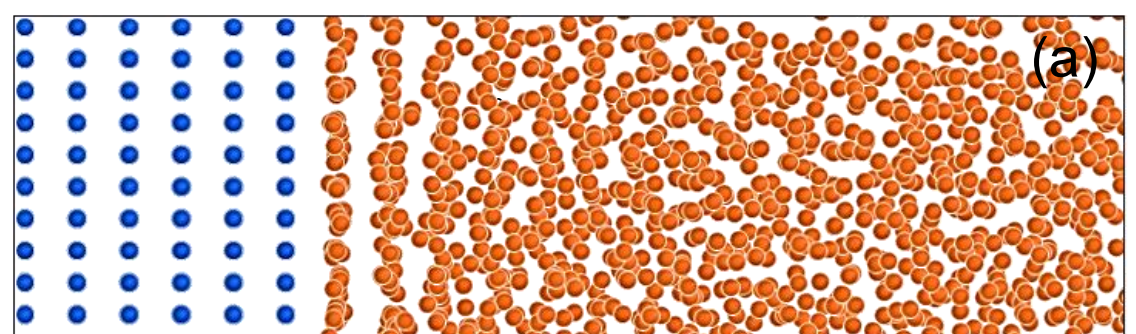


Fig. 2. (a) A snapshot of the simulation system of the liquid Al in contact with a crystalline Al substrate with a smooth surface equilibrated at $1000 \mathrm{~K}$. The liquid atoms adjacent to the liquid/substrate interface exhibit a layered structure and the quantified density profile, $\rho(z)$, is shown in (b).

Time-averaged atomic positions were used to characterize atomic arrangements of the liquid adjacent to the interface. Fig. 3 shows the time-averaged atomic positions of the $1^{\text {st }}, 2^{\text {nd }}, 3^{\text {rd }}$ and $4^{\text {th }}$ layers of the liquid at the interface corresponding to the simulation system in Fig. 2(a). The $1^{\text {st }}$ layer (Fig. 3(a)) exhibits a nearly ordered structure, and the atoms are largely confined to their equilibrium positions, which continue the lattice positions of the substrate surface. The $2^{\text {nd }}$ and $3^{\text {rd }}$ layers (Figs. 3(b\&c)) exhibit a mixed structure with ordered and disordered regions. The $4^{\text {th }}$ layer (Fig. 3(d)) displays a disordered structure, indicative of the characteristics of the liquid. This suggests that the ordered structure can extend from the substrate into the liquid by three atomic layers in the case of a smooth crystalline substrate $(R=0 \%)$. The atoms in the ordered regions of the $2^{\text {nd }}$ layer continue the lattice of the $1^{\text {st }}$ layer, and those in the $3^{\text {rd }}$ layer continue the lattice of the $2^{\text {nd }}$ layer. Therefore, the formation of ordered structure at the interface is achieved through the structural templating by the crystal lattice of the substrate surface.

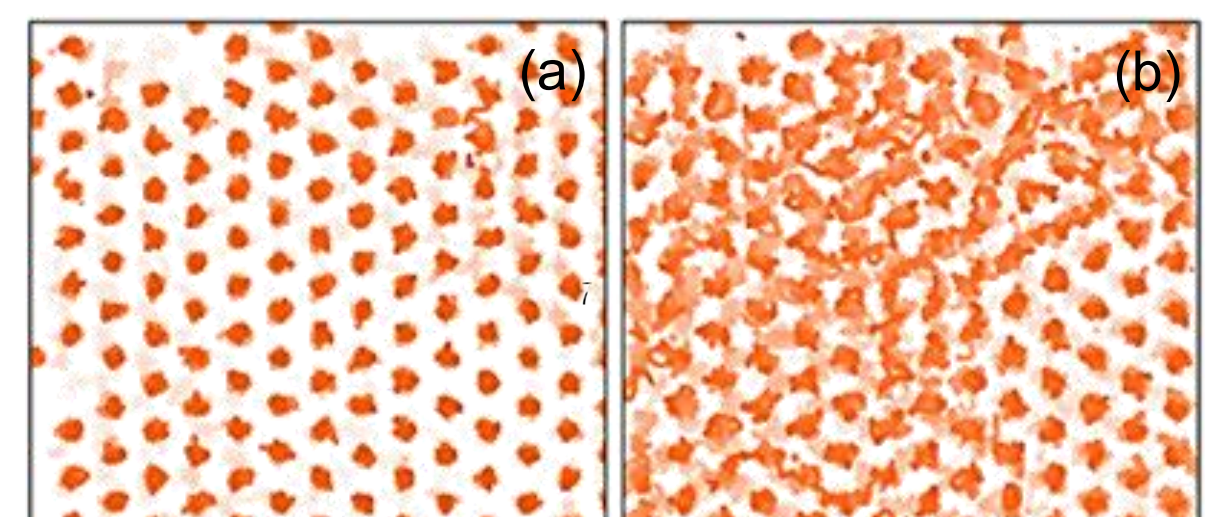



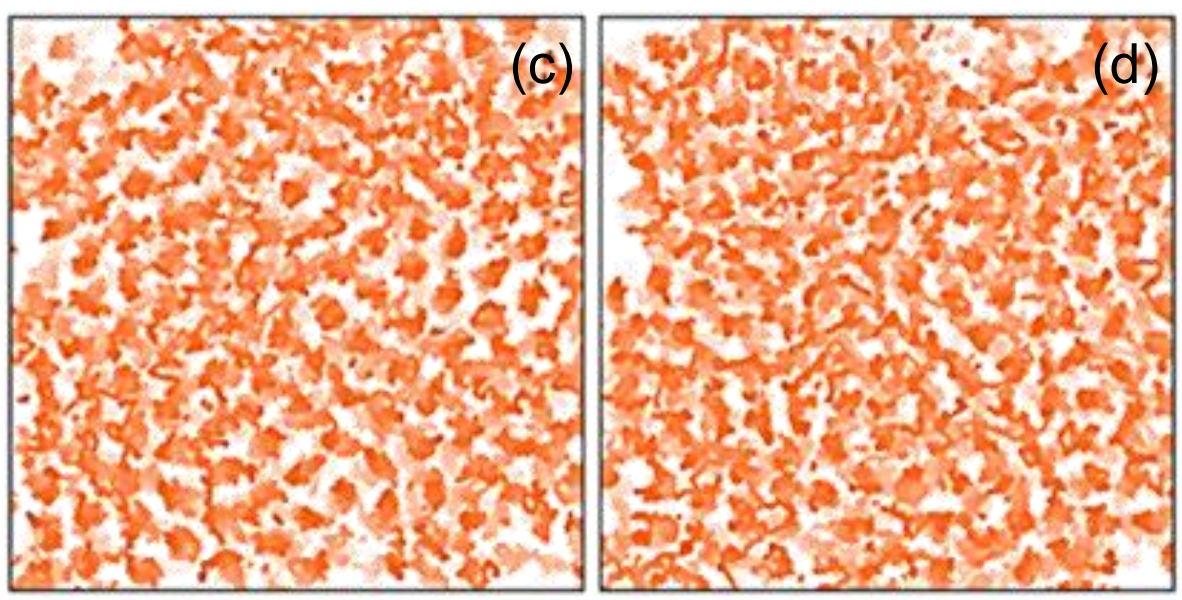

Fig. 3. Time-averaged atomic positions of the (a) $1^{\text {st }}$, (b) $2^{\text {nd }}$, (c) $3^{\text {rd }}$ and (d) $4^{\text {th }}$ atomic layers in the liquid adjacent to a smooth surface of the crystalline substrate during the simulation equilibrated at $1000 \mathrm{~K}$. The atoms exhibit an ordered structure in the $1^{\text {st }}$ layer, a mixed structure of ordered and disordered regions in the $2^{\text {nd }}$ and $3^{\text {rd }}$ layers, and a largely disordered structure in the $4^{\text {th }}$ layer.

Fig. 4 shows the density profiles of the liquid Al adjacent to crystalline substrates having different surface roughness $(R=0 \%, 20 \%$ and $40 \%)$, equilibrated at $1000 \mathrm{~K}$. Both the number of the layers and the peak height of the individual layer decrease with increasing substrate roughness. This suggests that a rough surface of a crystalline substrate impedes atomic ordering in the liquid adjacent to the interface. Therefore, it is necessary to investigate systematically the effect of surface roughness on atomic ordering in the liquid adjacent to the liquid/substrate interface.

Fig. 5(a) plots the peak density, $\rho_{\text {peak, }}$ of the first 4 layers in the liquid at the interface as a function of substrate roughness. The peak density of the $1^{\text {st }}$ layer decreases dramatically with increasing surface roughness of the crystalline substrate. For example, $\rho_{\text {peak }}$ of the $1^{\text {st }}$ layer is $0.36 \AA^{-3}$ for $R=9.3 \%$ and becomes $0.086 \AA^{-3}$ for $R=$ $98.9 \%$. The decrease in peak density becomes less dramatic for the subsequent layers. At large $R$ (e.g., $\geq 80 \%$ ), the value of $\rho_{\text {peak }}$ for the $1^{\text {st }}$ layer is only slightly larger than that for the $4^{\text {th }}$ layer, which is very close to that for the bulk liquid, suggesting that the layering adjacent to the interface becomes negligible with surfaces of high surface roughness.

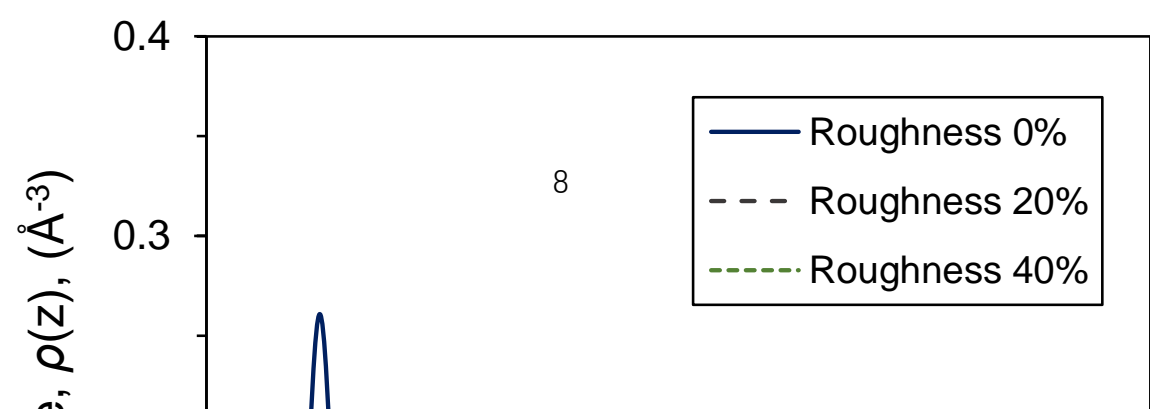


Fig. 4. Density profile, $\rho(\mathrm{z})$, of the liquid adjacent to crystalline substrates with varied surface roughness $(R)$ as a function of distance, $z$, from the interface, in the simulation equilibrated at $1000 \mathrm{~K}$. It shows that atomic layering at the interface degrades with increasing surface roughness of crystalline substrate.

The in-plane ordering of the liquid adjacent to the interface also deteriorates dramatically with increased substrate surface roughness, as shown in Fig. 5(b). Significant in-plane ordering exists within the first 2 atomic layers adjacent to the interface for small $R$, e.g., $R<30 \%$. With increasing $R$, the in-plane atomic ordering at the interface decreases substantially, for example, $S(z)$ of the $1^{\text {st }}$ layer is 0.79 for $R$ $=18 \%$ and decreases to 0.027 for $R=71 \%$. At large $R$, the in-plane atomic ordering becomes negligible even for the $1^{\text {st }}$ layer. This suggests that a rough crystalline surface can completely eliminate atomic ordering in the liquid adjacent to the liquid/substrate interface.

\subsection{Effect of surface roughness of crystalline substrates on atomic mobility}

We quantified the mobility of the atoms in the liquid at the interface by analyzing the frequency of the distribution of the atoms around their equilibrium positions in each individual layer, obtained from the time-averaged atomic positions.

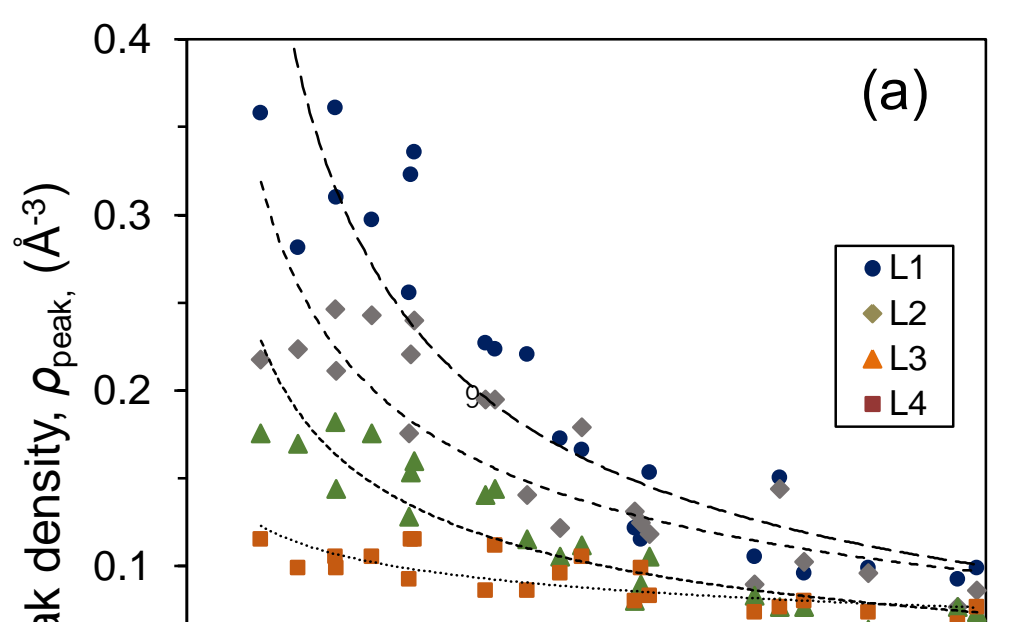




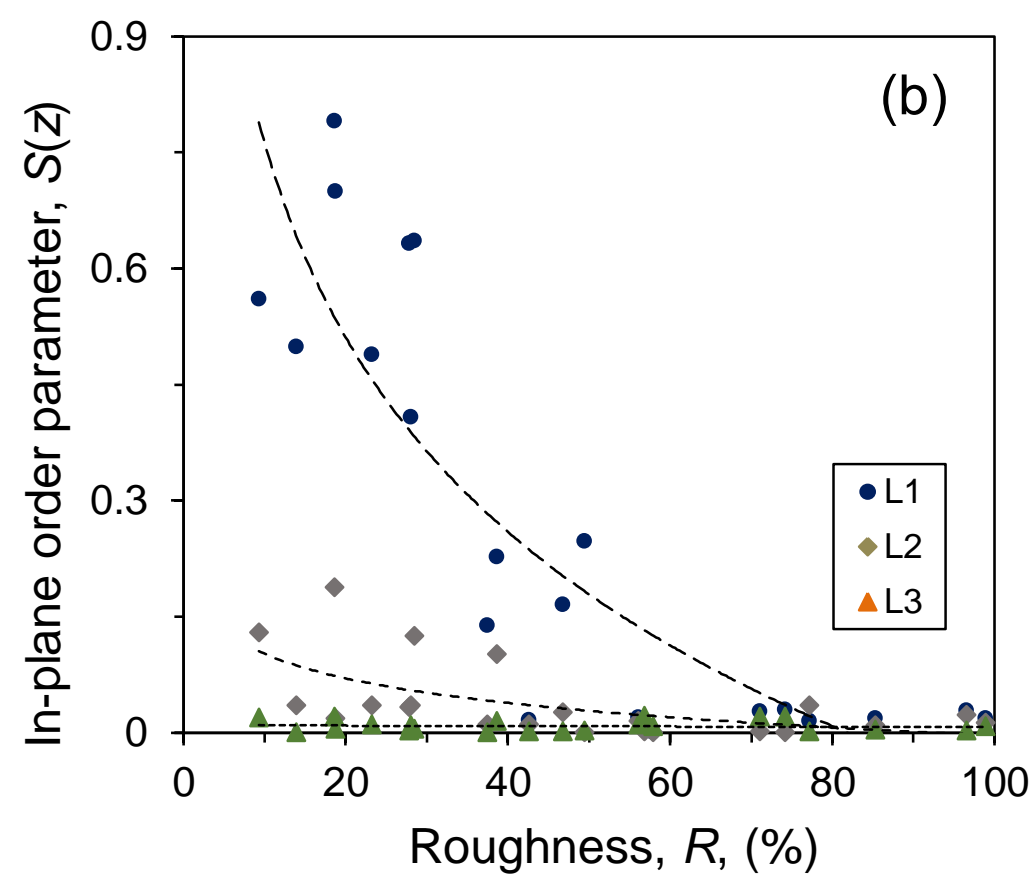

Fig. 5. (a) Peak density, $\rho_{\text {peak, }}$ and (b) in-plane order parameter, $S(z)$, of the $1^{\text {st }}, 2^{\text {nd }}, 3^{\text {rd }}$ and $4^{\text {th }}$ layers in the liquid adjacent to the crystalline substrate as a function of roughness, $R$, in the simulation equilibrated at $1000 \mathrm{~K}$. The dashed lines are the fitting for data of each layer. Both the layering and in-plane ordering adjacent to the interface deteriorate with increasing surface roughness of the crystalline substrate.

Fig. 6(a) shows the time-averaged atomic positions in the $1^{\text {st }}$ layer for 1000 time steps during the simulation for a system with a smooth crystalline surface equilibrated at 1000K. The circles in Fig. 6(a) represent distance, $x$, away from the equilibrium position of the individual atom in the corresponding layer. The atoms in the $1^{\text {st }}$ layer are largely localized to their equilibrium position provided by the lattice of the substrate surface. The frequency distribution at $x$ for each atom, $f(x)$, can be obtained by calculating the ratio of the counts that the atom enters the region between the consecutive circles in unit time. The average frequency for all atoms in the $1^{\text {st }}$ layer as a function of $x$ is plotted in Fig. 6(b). The value of $f(x)$ decreases with increasing $|x|$ from the equilibrium position, and the data of $f(x)$ can be well fitted by a normal 
distribution function, as exhibited in Fig. 6(b). The full width at half maximum (FWHM) of the fitted curve indicates the degree of the deviation of atoms from their equilibrium positions, and hence can be used as a measure of the mobility of atoms in the corresponding layers at the interface.

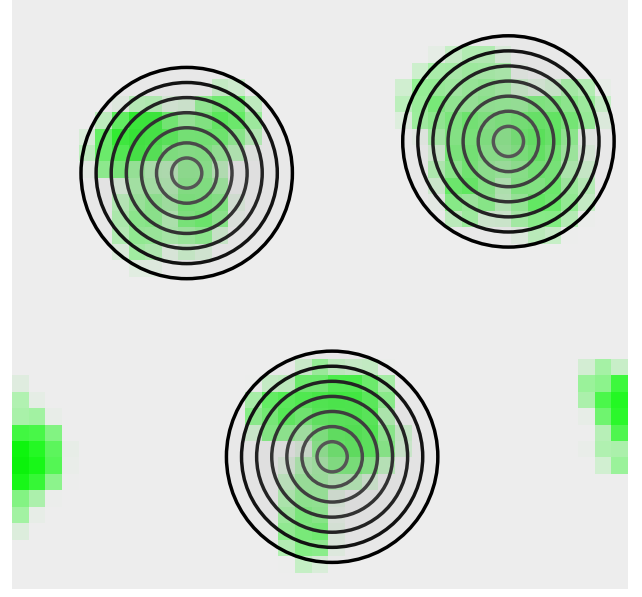

(a)

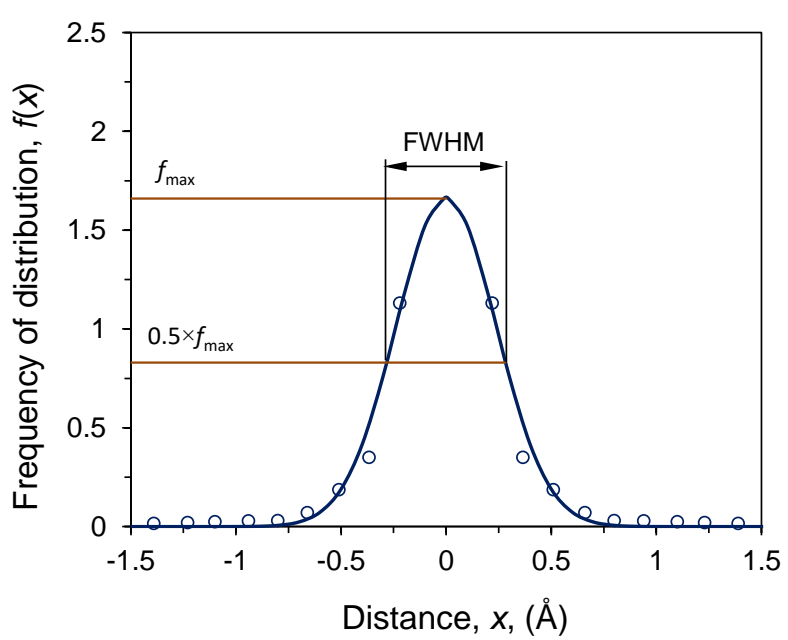

(b)

Fig. 6. (a) Time-averaged atomic positions in part of the $1^{\text {st }}$ layer of the liquid adjacent to a smooth crystalline substrate for 1000 time steps in the simulation equilibrated at $1000 \mathrm{~K}$; and (b) frequency distribution, $f(x)$, of an individual atom in the $1^{\text {st }}$ layer of the liquid as a function of distance, $x$, away from its equilibrium position. The circles in (a) represent the distances from the equilibrium position. The open circles in (b) represent the quantified frequency data, and the solid line is the fitting of a normal distribution for the frequency of the distribution, where FWHM (full width at half maximum) has been taken as a measure of the mobility of this atom.

Fig. 7(a) shows the frequency distribution of the atoms in the first 4 layers in the liquid adjacent to the interface as a function of distance $(x)$ from the equilibrium positions for the simulation system with a smooth crystalline surface equilibrated at 1000K. The fitting curves in Fig. 7(a) represent normal distributions. The distribution curve is sharp for the $1^{\text {st }}$ layer, with a small FWHM and a large peak, and becomes increasingly wider for the subsequent layers, with a decreasing peak. This result implies that the average mobility of the atoms increases with increasing the distance from the interface. It is noted that the distribution curves for the $3^{\text {rd }}$ and $4^{\text {th }}$ layers are almost identical, suggesting that the atoms in the $3^{\text {rd }}$ layer and beyond have the same atomic mobility as the bulk liquid. The average FWHM of the atoms in the $1^{\text {st }}$ layer was calculated for the simulation system with crystalline substrates having varied surface roughness, and the results are presented in Fig. 7(b) as a function of the surface roughness. The FWHM increases with increasing $R$, suggesting that the mobility of the atoms in the $1^{\text {st }}$ layer increases with increasing surface roughness of the crystalline substrate. 

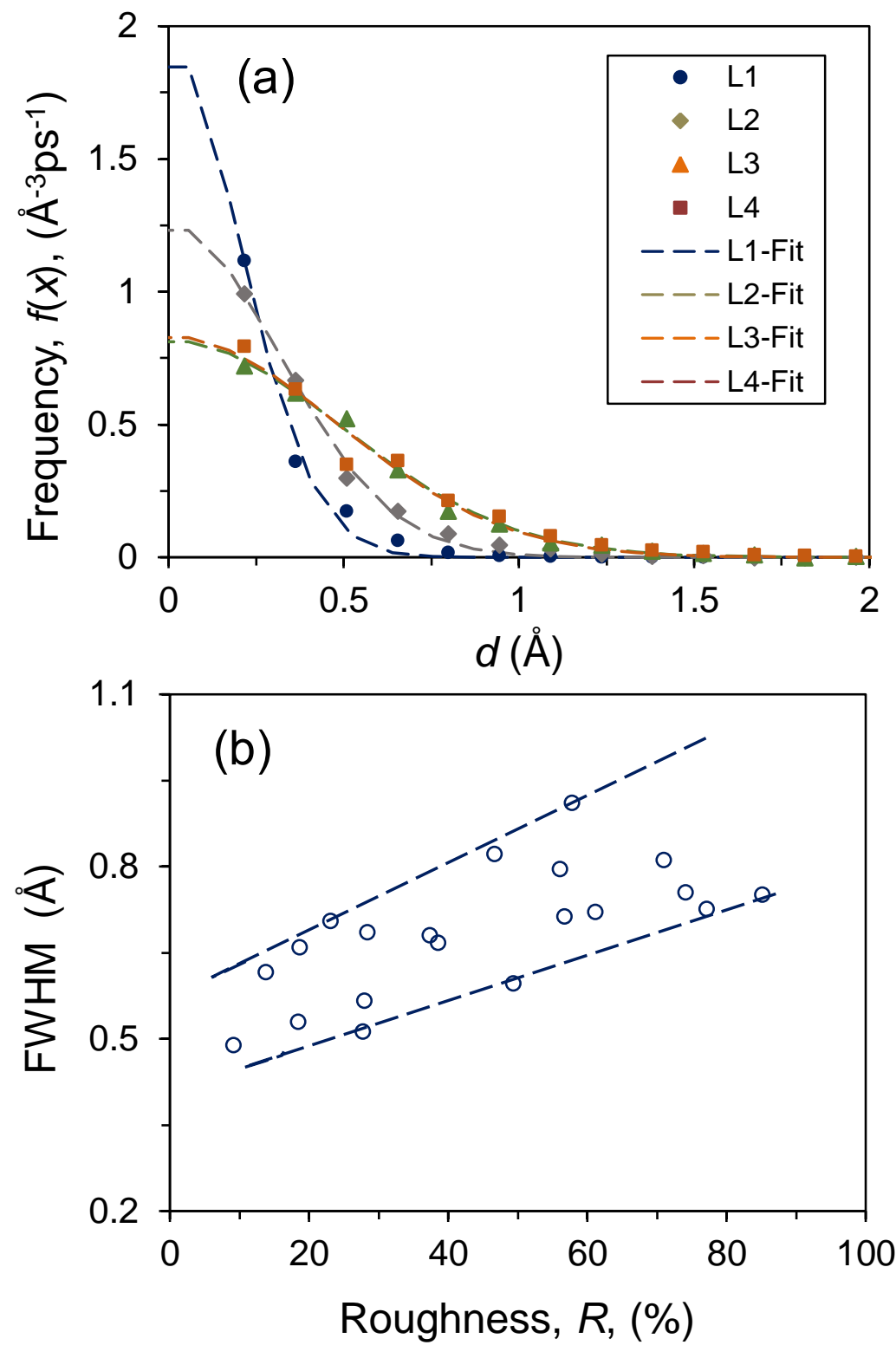

Fig. 7. (a) Averaged frequency distribution of atoms in the $1^{\text {st }}, 2^{\text {nd }}, 3^{\text {rd }}$ and $4^{\text {th }}$ layers of the liquid adjacent to a smooth crystalline substrate as a function of the distance, $x$, from the equilibrium position; and (b) FWHM of the frequency distribution in the $1^{\text {st }}$ layer as a function of surface roughness for 1000 time steps in the simulation equilibrated at $1000 \mathrm{~K}$. The dashed lines mark the fitting of normal distributions in (a), and the envelope of scattered data of FWHM in (b).

\subsection{Effect of surface roughness of amorphous substrates on atomic ordering}

The atomic ordering in the liquid at the interface disappears completely for the simulation system with a bulk amorphous substrate, which has a surface roughness of $R=100 \%$. There is no layered structure at the interface observed in the snapshot of the simulation system equilibrated at $1000 \mathrm{~K}$ (Fig. 8(a)). The density profile, $\rho(\mathrm{z})$, of the liquid at the interface does not show the usual oscillation exhibited by the systems with a smooth substrate (Fig. 8(b)). This suggests that the rough surface of an 
amorphous substrate eliminates the atomic layering in the liquid adjacent to the interface. Fig. 9 shows the time-averaged atomic positions of the $1^{\text {st }}, 2^{\text {nd }}, 3^{\text {rd }}$ and $4^{\text {th }}$ layers at the interface. Some of the liquid atoms entered the surface layer of the amorphous substrate (see Fig. 8(a)), and therefore the $1^{\text {st }}$ layer of the liquid is not fully filled by liquid atoms (see Fig. 9(a)). All the layers at the interface exhibit a disordered structure, indicating that atoms in the liquid adjacent to a bulk amorphous substrate have the same characteristics as the bulk liquid. Therefore, it can be concluded that a bulk amorphous substrate eliminates atomic ordering in the liquid at the interface; neither atomic layering nor in-plane atomic ordering exist in the liquid adjacent to the interface.

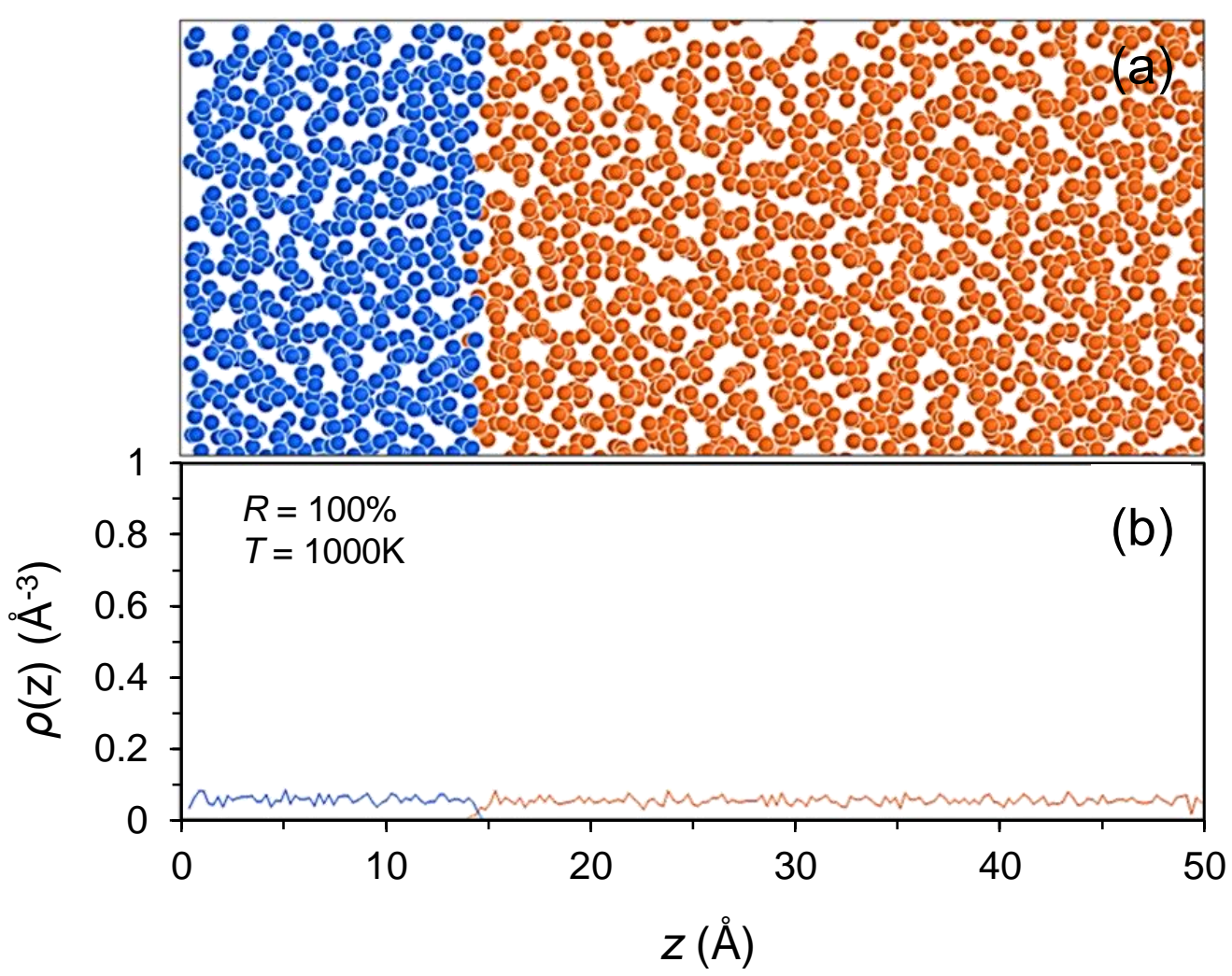

Fig. 8. (a) A snapshot and (b) density profiles, $\rho(z)$, of the liquid atoms adjacent to the bulk amorphous substrate with a rough surface in the simulation equilibrated at $1000 \mathrm{~K}$. Atomic layering is negligible adjacent to the interface.
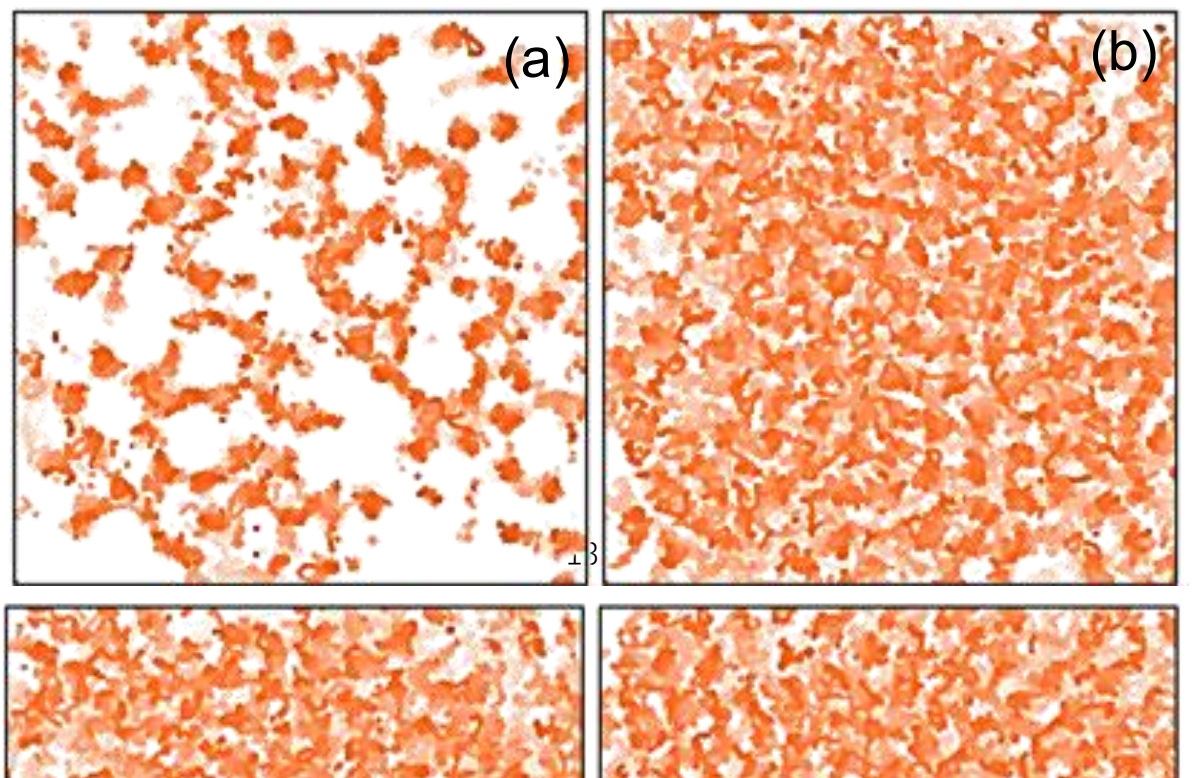
(a)

Fig. 9. Time-averaged atomic positions of the (a) $1^{\text {st }}$, (b) $2^{\text {nd }}$, (c) $3^{\text {rd }}$ and (d) $4^{\text {th }}$ atomic layers in the liquid adjacent to the rough surface of a bulk amorphous substrate in the simulation equilibrated at $1000 \mathrm{~K}$. The atoms exhibit a disordered structure at and adjacent to the interface.

Fig. 10 shows the peak density and in-plane order parameter of the individual layers in the liquid adjacent to the interface as a function of distance $z$ from the interface for the simulation system with amorphous substrates having varied surface roughness. Since the atomic positions of the $1^{\text {st }}$ layer were only partially occupied by the liquid atoms, the $1^{\text {st }}$ layer consequently has a low $\rho_{\text {peak, }}$ as shown in Fig. 10(a). From the $2^{\text {nd }}$ layer onwards, $\rho_{\text {peak }}$ of the corresponding layers at the interface decreases with increasing $z$ from the interface. Atomic layering decreases with increasing surface roughness, in terms of number of layers and the corresponding peak density. These results suggest that atomic layering in the liquid is strong for the systems with a smooth amorphous substrate ( $R=0 \%$, not shown here), and decreases sharply with increasing surface roughness.

The simulation results for the in-plane order parameter are presented in Fig. 10(b), which suggests that no in-plane atomic ordering exists adjacent to the interface with an amorphous substrate, regardless of surface roughness. The in-plane order parameter, $S(z)$, does not show any obvious variation with $R$, and the value of $S(z)$ is below 0.01, which is close to that of the bulk liquid. It can be concluded from Fig. 10 that the atomic layering in the liquid at the interface is impeded substantially by increasing surface roughness of an amorphous substrate whilst the in-plane atomic ordering is eliminated completely by an amorphous substrate regardless of surface roughness.

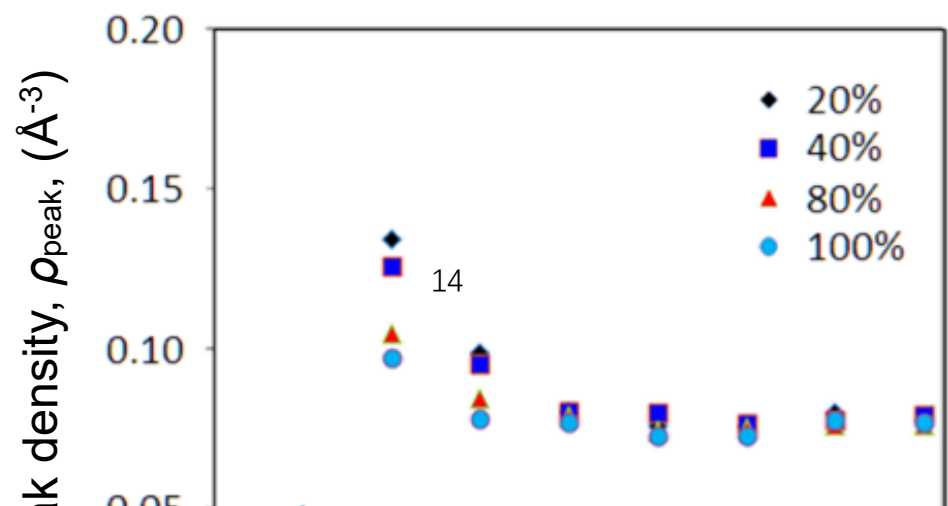




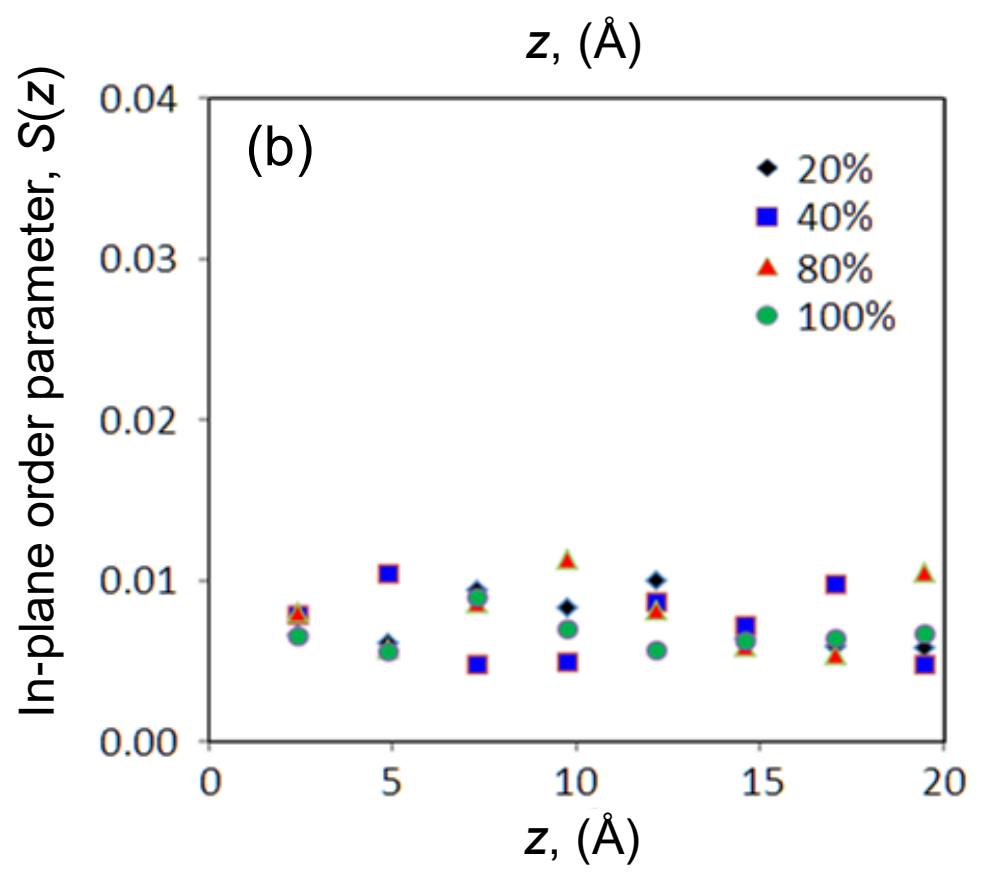

Fig.10. (a) Peak density, $\rho_{\text {peak, }}$ and (b) in-plane order parameter, $S(z)$, of the liquid adjacent to the interface with a single layer of an amorphous substrate with varied roughness as a function of distance, $z$, from the interface, in the simulation equilibrated at $1000 \mathrm{~K}$. The $1^{\text {st }}$ layer at the interface has a relatively low peak density, which accounts for the liquid atoms occupying a fraction of atomic positions in surface layer of the substrate.

\section{Discussion}

In this work we have adopted a number of unique approaches to investigate the effect of atomic level surface roughness on atomic ordering in the liquid at the liquid/substrate interface. Firstly, the substrate atoms are chosen to be the same as those of the liquid in terms of chemical nature, and have a lattice parameter equal to that of the solid phase (Al) at its melting point. By doing so, we can eliminate the effects of both chemical interaction between the substrate and the liquid and the lattice misfit between the substrate and the solid phase, which have been confirmed to have a significant effect on atomic ordering in the liquid adjacent to the interface $[3,22]$. 
Secondly, in all MD simulations, the atoms of the substrates, both crystalline and amorphous, are pinned at their equilibrium positions. This allows the simulation of substrates with different melting temperatures [22]. In addition, it has been confirmed that MD simulation with pinned substrates increases only slightly the atomic ordering in the liquid adjacent to the interface compared with that with a relaxed substrate, and that such minor difference would not affect the overall trends of atomic ordering [22]. Thirdly, we constructed artificially atomic level rough surfaces with varying surface roughness for both crystalline and amorphous substrates. Although such artificial rough surfaces may not be directly related to any rough surface in reality, it does allow us to investigate systematically the effect of surface roughness on atomic ordering in the liquid. Fourthly, we use the FWHM (full width at half maximum) of the atomic position distributions to quantify atomic mobility in the liquid at the interface, which allows us to connect atomic ordering in the liquid at the interface with surface roughness. Finally, for the first time we have defined atomic level surface roughness (Eq. (1)), which will facilitate further scientific investigation into atomic level surface roughness. It should be pointed out that atomic level rough surface is different from those at the nano- or micro-scale [29-34]. A rough surface at the microscopic scale may become smooth at the atomic level due to the existence of facets at the atomic level. Such microscopic rough surfaces may enhance atomic ordering [31,33]. Overall, these unique approaches to MD simulation allow the effect of surface roughness of the substrate to be assessed systematically.

It is noticed that there is considerable scatter of data for both the peak density (Fig. 5(a)) and the in-plane order parameter (Fig. 5(b)). More detailed data analysis suggests that such scattering is caused by the artificial creation of the rough substrate surface. With the surface roughness being defined by Eq. (2), a given surface roughness, $R$, can be achieved by different combinations of $p$ and $\alpha$ (see Fig. 1(a)), among which larger $p$ and smaller $\alpha$ favour higher values for both peak density and in-plane order parameter. This is because larger $p$ gives rise to smooth platelets, which in turn induce local atomic ordering normal to the platelets, and consequently resulting in higher values for peak density and in-plane order parameter normal to the rough surface. However, it should be pointed out that such data scattering in Fig. 5 does not affect the general conclusion that atomic ordering in the liquid at the interface is reduced by increasing atomic level surface roughness.

In this study, we have found for the first time that the surface roughness of a crystalline substrate reduces both atomic layering and in-plane atomic ordering in the metallic liquid adjacent to the interface. In addition, we have also found that the rough surface of an amorphous substrate completely eliminates in-plane ordering in the liquid regardless of surface roughness, and reduces/eliminates atomic layering in the liquid depending on the surface roughness. This provides further advance on our understanding of atomic ordering in the liquid induced by a solid substrate. Our previous MD simulations [14] have revealed that a smooth crystalline surface can 
induce significant atomic ordering in the liquid adjacent to the substrate, which is manifested by atomic layering normal to the interface and in-plane atomic ordering parallel to the interface. More specifically, the atomic layering is independent of lattice misfit between the substrate and the solidified phase, suggesting that atomic layering is largely a geometrical effect of the smooth substrate surface, i.e., the "hard wall" effect [17]; however, the in-plane ordering is strongly dependent on the lattice misfit and can be attributed to structural templating, in which liquid atoms become ordered when they occupy the low energy positions provided by the crystalline lattice beneath them. In this study, we found that the mobility of the liquid atoms adjacent to a rough crystalline surface increases with increasing surface roughness (Fig. 7). This increased atomic mobility impedes both the "hard wall" effect for atomic layering and the structural templating for the in-plane atomic ordering, resulting in a reduced atomic ordering in the liquid with increasing surface roughness of the substrate (Fig. 5). In the case of amorphous substrates, the condition for structural templating is completely destroyed by a structureless rough surface, therefore eliminating the in-plane atomic ordering in the liquid. However, although the atomic layering in such cases is significantly reduced by increasing the surface roughness of the amorphous substrate, it can exist when the surface roughness is small. It should be pointed out that the level of atomic layering would be the same for both amorphous and crystalline surfaces as long as the substrate surface is smooth $(R=0 \%)$.

From the point of view of heterogeneous nucleation, atomic ordering in the liquid adjacent to the liquid/substrate interface at temperatures above the liquidus has been referred to as the prenucleation phenomenon [3]. From previous studies we understand that prenucleation can be enhanced by reducing the lattice misfit between the substrate and the solid through structural templating [3], which can be further enhanced by choosing a substrate that has attractive chemical interaction with the liquid atoms, i.e., having a large and negative heat of mixing [22]. On the other hand, prenucleation can be impeded effectively by choosing a substrate of large lattice misfit, and/or of a large and positive heat of mixing with the liquid. Based on the MD simulation results in this work, one expects that prenucleation can also be impeded by choosing a substrate of atomic level surface roughness. In addition, the present work provides a further confirmation of the importance of structural templating in heterogeneous nucleation. A crystalline substrate with an atomic level rough surface would have a reduced potency for heterogeneous nucleation; and an amorphous substrate with a rough surface or a structureless smooth surface would be impotent for heterogeneous nucleation since it would be less competitive for heterogeneous nucleation with other crystalline substrates available in the liquid.

\section{Summary}

In this study, we investigated systematically the effect of atomic level surface roughness of both crystalline and amorphous substrates on atomic ordering in the 
liquid adjacent to the liquid/substrate interface, using molecular dynamics simulation. The atomic ordering at the interface was characterized by the atomic layering, in-plane atomic ordering and the time-averaged atomic positions, and the mobility of the atoms was quantified by the full width at half maximum (FWHM) of the frequency distribution of the individual atoms in each layer adjacent to the interface. We have found for the first time that surface roughness of a crystalline substrate reduces both atomic layering and in-plane atomic ordering in the metallic liquid adjacent to the interface. In addition, we have also found that the roughness of the surface of an amorphous substrate completely eliminates in-plane ordering in the liquid regardless of surface roughness and reduces/eliminates atomic layering in the liquid depending on the surface roughness. This reduced atomic ordering in the liquid adjacent to an atomically rough surface can be attributed to the increase in mobility of atoms in the liquid. This significantly advances our understanding of atomic ordering in the liquid induced by a solid substrate. In addition, from the point of view of the heterogeneous nucleation, this work provides further confirmation of the importance of structural templating as a mechanism for both prenucleation and heterogeneous nucleation. Furthermore, this work provides a new approach to impede heterogeneous nucleation by roughening the substrate surface.

\section{Acknowledgement}

EPSRC is gratefully acknowledged for providing financial support under Grant $\mathrm{EP} / \mathrm{H} 026177 / 1$.

\section{Declarations of interest}

None

\section{Data availability}

The raw/processed data required to reproduce these findings cannot be shared at this time due to technical or time limitations.

\section{References}

[1] W.D. Kaplan, Y. Kauffmann, Structural order in liquids induced by interfaces with crystals, Annu. Rev. Mater. Res. 36 (2006) 1-48.

[2] A.L. Greer, Liquid metals supercool order, Nature Mater. 5 (2006) 13-14.

[3] H. Men, Z. Fan, Prenucleation induced by crystalline substrates, Matell. Mater. Trans. A, (in revision, 2018).

[4] Z. Fan, An epitaxial model for heterogeneous nucleation on potent substrates, Metall. Mater. Trans. A 44 (2013) 1409-1418.

[5] W.J. Huisman, J.F. Peters, M.J. Zwanenburg, S.A. de Vries, T.E. Derry, D. 
Abernathy, J.F. van der Veen, Layering of a liquid metal in contact with a hard wall, Nature 390 (1997) 379-381.

[6] H. Reichert, O. Klein, H. Dosch, M. Denk, V. Honkimäki, T. Lippmann, G. Reiter, Observation of five-fold local symmetry in liquid lead, Nature 408 (2000) 839-841.

[7] A.K. Doerr, M. Tolan, J.P. Schlomka, W. Press, Evidence for density anomalies of liquids at the solid/liquid interface, Euro. Phys. Lett. 52 (2000) 330-336.

[8] S.E. Donnelly, R.C. Birtcher, C.W. Allen, I. Morrison, K. Furuya, M.H. Song, K. Mitsuishi, U. Dahmen, Ordering in a fluid inert gas confined by flat surfaces, Science 296 (2002) 507-510.

[9] S.H. Oh, Y. Kauffmann, C. Scheu, W.D. Kaplan, M. Rühle, Ordered liquid aluminium at the interface with sapphire, Science 310 (2005) 661-663.

[10] S.H. Oh, C. Scheu, M. Rühle, In-situ HRTEM studies of alumina-aluminium solid-liquid interfaces, Korean J. Electron Microscopy Special Issue 1 (2006) 19-24.

[11] P. Geysermans, D. Gorse, V. Pontikis, Molecular dynamics study of the solid-liquid interface, J. Chem. Phys. 113 (2000) 6382-6389.

[12] A. Hashibon, J. Adler, M.W. Finnis, W.D. Kaplan, Ordering at solid-liquid interfaces between dissimilar materials, Interface Sci. 9 (2001) 175-181.

[13] A. Hashibon, J. Adler, M.W. Finnis, W.D. Kaplan, Atomistic study of structural correlations at a liquid-solid interface, Comp. Mater. Sci. 24 (2002) 443-452.

[14] H. Men, Z. Fan, Atomic ordering in liquid aluminium induced by substrates with misfits, Comp. Mater. Sci. 85 (2014) 1-7.

[15] J.S. Wang, A. Horsfield, U. Schwingenschlögl, P.D. Lee, Heterogeneous nucleation of solid $\mathrm{Al}$ from the melt by $\mathrm{TiB}_{2}$ and $\mathrm{Al}_{3} \mathrm{Ti}$ : $\mathrm{An}$ ab initio molecular dynamics study, Phys. Rev. B 82 (2010) 184203.

[16] J.S. Wang, A. Horsfield, P.D. Lee, P. Brommer, Heterogeneous nucleation of solid Al from the melt by $\mathrm{Al}_{3} \mathrm{Ti}$ : Molecular dynamics simulations, Phys. Rev. B 82 (2010) 144203.

[17] M.J. Regan, E.H. Kawamoto, S. Lee, P.S. Pershan, N. Maskil, M. Deutsch, O.M. Magnussen, B.M. Ocko, L.E. Berman, Surface layering in liquid gallium: An X-ray reflectivity study, Phys. Rev. Lett. 75 (1995) 2498-2501.

[18] J. Fischer, M. Methfessel, Born-Green-Yvon approach to the local densities of a fluid at interfaces, Phys. Rev. A 22 (1980) 2836-2843.

[19] O.M. Magnussen, B.M. Ocko, M.J. Regan, K. Penanen, P.S. Pershan, M. Deutsch, $\mathrm{X}$-ray reflectivity measurements of surface layering in liquid mercury, Phys. Rev. Lett. 74 (1995) 4444-4447.

[20] O.G. Shpyrko, A.Y. Grigoriev, C. Steimer, P.S. Pershan, B. Lin, M. Meron, T. Graber, J. Gerbhardt, B. Ocko, M. Deutsch, Anomalous layering at the liquid Sn surface, Phys. Rev. B 70 (2004) 224206.

[21] C.J. Yu, A.G. Richter, A. Datta, M.K. Durbin, P. Dutta, Observation of molecular layering in thin liquid films using X-ray reflectivity, Phys. Rev. Lett. 82 (1999) 2326-2329. 
[22] C.M. Fang, H. Men, Z. Fan, to be submitted (2018).

[23] T.M. Galea, P. Attard, Molecular dynamics study of the effect of atomic roughness on the slip length at the fluid-solid boundary during shear flow, Langmuir, 20 (2004) 3477-3482.

[24] V. Rosato, M. Guillope, B. Legrand, Thermodynamical and structural properties of f.c.c. transition metals using a simple tight-binding model, Phil. Mag. A 59 (1989) 321-336.

[25] C. Kittel, Introduction to solid state physics, Wiley-Interscience, New York, 1996.

[26] M.W. Chase, J. Phys. Chem. Ref. Data, Monograph 9, NIST-JANAF Thermochemical Tables, $4^{\text {th }}$ ed., Part I, National Institute of Standard Technology, Gaithersburg, Maryland, 1998, pp. 61.

[27] G. Simons, H. Wang, Single crystal elastic constants and calculated aggregate properties, MIT, Cambridge, MA, 1977.

[28] J.R. Hook, H.E. Hall, Solid state physics, $2^{\text {nd }}$ ed., Wiley, Chichester, 1991.

[29] C. Cottin-Bizonnea, C. Barentin, E. Charlaix, L. Bocquetb, J.-L. Barrat, Dynamics of simple liquids at heterogeneous surfaces: Molecular-dynamics simulations and hydrodynamic description, Eur. Phys. J. E 15 (2004) 427-438.

[30] D.J. Kim, E. Darve, Molecular dynamics simulation of electro-osmotic flows in rough wall nanochannels, Phys. Rev. E 73 (2006) 051203.

[31] A.J. Page, R.P. Sear, Heterogeneous nucleation in and out of pores, Phys. Rev. Lett. 97 (2006) 065701.

[32] R.P. Sear, Nucleation: theory and applications to protein solutions and colloidal suspensions, J. Phys.: Condens. Mat. 19 (2007) 033101.

[33] R. Kamali, A. Kharazmi, Molecular dynamics simulation of surface roughness effects on nanoscale flows, Int. J. Thermal Sci. 50 (2011) 226e232.

[34] Z. Jiao, C.B. Song, T.S. Lin, P. He, Molecular dynamics simulation of the effect of surface roughness and pore on linear friction welding between $\mathrm{Ni}$ and $\mathrm{Al}$, Comput. Mater. Sci. 50 (2011) 3385-3389. 